\title{
Roles of Three Domains of Tetrahymena eEF1A in Bundling F-actin
}

\author{
Kenya Morita, Fumihide Bunai and Osamu Numata* \\ Graduate School of Life and Environmental Sciences, University of Tsukuba, \\ Tsukuba, Ibaraki 305-8572, Japan
}

\begin{abstract}
The conventional role of eukaryotic elongation factor 1A (eEF1A) is to transport aminoacyl tRNA to the $A$ site of ribosomes during the peptide elongation phase of protein synthesis. eEF1A also is involved in regulating the dynamics of microtubules and actin filaments in cytoplasm. In Tetrahymena, eEF1A forms homodimers and bundles $\mathrm{F}$-actin. $\mathrm{Ca}^{2+} /$ calmodulin (CaM) causes reversion of the eEF1A dimer to the monomer, which loosens F-actin bundling, and then $\mathrm{Ca}^{2+} / \mathrm{CaM} / \mathrm{eEF} 1 \mathrm{~A}$ monomer complexes dissociate from F-actin. eEF1A consists of three domains in all eukaryotic species, but the individual roles of the Tetrahymena eEF1A domains in bundling F-actin are unknown. In this study, we investigated the interaction of each domain with F-actin, recombinant Tetrahymena CaM, and eEF1A itself in vitro, using three glutathione-S-transferase-domain fusion proteins (GST-dm1, -2 , and -3 ). We found that only GST-dm3 bound to F-actin and influences dimer formation, but that all three domains bound to Tetrahymena $\mathrm{CaM}$ in a $\mathrm{Ca}^{2+}$-dependent manner. The critical $\mathrm{Ca}^{2+}$ concentration for binding among three domains of eEF1A and CaM were $\leq 100 \mathrm{nM}$ for domain 1, 100 $\mathrm{nM}$ to $1 \mu \mathrm{M}$ for domain 3 , and $>1 \mu \mathrm{M}$ for domain 2 , whereas stimulation of and subsequent $\mathrm{Ca}^{2+}$ influx through $\mathrm{Ca}^{2+}$ channels raise the cellular $\mathrm{Ca}^{2+}$ concentration from the basal level of $\sim 100 \mathrm{nM}$ to $\sim 10 \mu \mathrm{M}$, suggesting that domain 3 has a pivotal role in $\mathrm{Ca}^{2+} / \mathrm{CaM}$ regulation of eEF1A.
\end{abstract}

Key words: Tetrahymena, eukaryotic translation elongation factor $1 \mathrm{~A}(\mathrm{eEF} 1 \mathrm{~A})$, actin, cytoskeleton, $\mathrm{Ca}^{2+}$, calmodulin

\section{INTRODUCTION}

Eukaryotic elongation factor $1 \mathrm{~A}$ (eEF1A; formerly EF$1 \alpha$; EF1A in prokaryotes) is an abundant cytoplasmic protein that is conserved in all eukaryotes. It delivers aminoacyltRNA (aa-tRNA) to the A site of ribosomes during the peptide elongation phase of protein synthesis. Binding of eEF1A with the eEF1B guanine nucleotide exchange factor (GEF) complex leads to replacement of GDP with GTP, and on binding GTP, eEF1A can interact with aa-tRNA. Subsequently, the binding of eEF1A/GTP/aa-tRNA ternary complex with the ribosome triggers the GTPase activity of eEF1A and subsequent dissociation of eEF1A/GDP from the ribosome. Structural data show that EF1A and eEF1A consist of three domains (Andersen et al., 2000, 2003), which are well conserved among species. Domain 1 is composed of about 200 amino acid residues and has a central $\beta$ sheet surrounded by eight $\alpha$ helices. The active site of the GTPase activity of eEF1A, the G domain, is in domain 1 . Domains 2 and 3 each contain about 100 amino acid residues and have $\beta$ barrels.

In addition to its role in translation elongation, eEF1A is involved in the regulation of microtubules and the actin cytoskeleton. In carrot, a homolog of eEF1A has microtu-

\footnotetext{
* Corresponding author. Phone: +81-29-853-6648; Fax : +81-29-853-6614; E-mail : numata@sakura.cc.tsukuba.ac.jp
}

bule-bundling activity that is regulated by a $\mathrm{Ca}^{2+} /$ calmodulin (CaM)-sensitive mechanism (Durso and Cry, 1994). The multifunctional eEF1A protein also participates in F-actin bundling. The actin-binding and F-actin-bundling activities of eEF1A were demonstrated initially in Dictyostelium (Yang et al., 1990), and these functions of eEF1A are retained in other species, such as Physarum polycephalum (Itano and Hatano, 1991), Tetrahymena (Kurasawa et al., 1996a), and fission yeast (Suda et al., 1999). Because eEF1A binds to F-actin with relatively high affinity, eEF1A may be a potent regulator of the dynamics of the actin cytoskeleton. In Dictyostelium, binding between eEF1A and actin is sensitive to cellular parameters such as nucleotide concentration and pH (Edmonds et al., 1995, 1998; Liu et al., 1996). In vitro analysis of Dictyostelium eEF1A showed that domains 1 and 3 of eEF1A bind to F-actin, suggesting that eEF1A monomer can bundle F-actin (Liu et al., 2002). In addition, Dictyostelium eEF1A has a role in anchoring $\beta$-actin mRNA to the actin cytoskeleton (Liu et al., 2002).

Tetrahymena eEF1A bundles rabbit skeletal muscle $\mathrm{F}$ actin in addition to Tetrahymena F-actin (Kurasawa et al., 1996b). Using Tetrahymena eEF1A, Tetrahymena actin, and recombinant Tetrahymena CaM, Kurasawa et al. (1996a) demonstrated that $\mathrm{Ca}^{2+} / \mathrm{CaM}$ bound to eEF1A and completely inhibited subsequent formation of F-actin bundles. Furthermore, Bunai et al. (2006) demonstrated that Tetrahymena eEF1A dimerized and bundled F-actin but eEF1A monomer did not. $\mathrm{Ca}^{2+} / \mathrm{CaM}$ separated eEF1A dimers into monomers, thereby decreasing the affinity of 
eEF1A for F-actin; $\mathrm{Ca}^{2+} / \mathrm{CaM} / \mathrm{eEF} 1 \mathrm{~A}$ monomer complexes dissociated from F-actin. Conversely, chelation of $\mathrm{Ca}^{2+}$ by eEF1A monomer led to dimer formation (Bunai et al., 2006). Therefore, the F-actin bundling activity of eEF1A is reversibly regulated by $\mathrm{Ca}^{2+} / \mathrm{CaM}$.

In Tetrahymena, the roles of the three eEF1A domains in F-actin bundling are unknown. To understand the roles of these domains in dimer formation and the interaction of Tetrahymena eEF1A with $\mathrm{F}$-actin and $\mathrm{Ca}^{2+} / \mathrm{CaM}$, we prepared GST fusion proteins with domains 1, 2, and 3 in Escherichia coli. We then investigated the interaction of these three GST-domain fusion proteins (GST-dm1, -2, and -3) with F-actin, recombinant Tetrahymena CaM, and eEF1A in vitro. Domain 3 is responsible for forming dimers and binding F-actin, and all three domains bind CaM.

\section{MATERIALS AND METHODS}

\section{Cell culture}

Tetrahymena pyriformis (strain W) was cultivated in PYD medium ( $1 \%$ proteose peptone, $0.5 \%$ yeast extract, $0.87 \% \mathrm{D}$ glucose) at $26^{\circ} \mathrm{C}$ (Watanabe et al., 1994).

\section{Preparation of Tetrahymena eEF1A and rabbit skeletal muscle actin}

Tetrahymena eEF1A was purified as described previously (Takeda et al., 1995). Rabbit skeletal muscle actin was purified according to the procedure of Pardee and Spudich (1982).

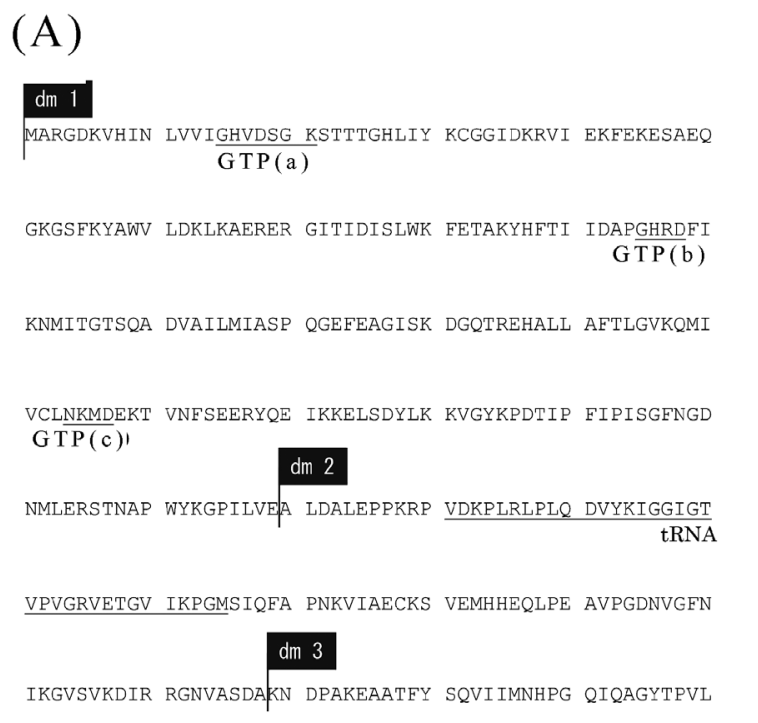

DCHTAHIACK FETIHDKIDR RTGKSQEENP KFIKNGDAAL VTLIPTKALC

VEVEQEYPPL GRYAVRDMKQ TVAVGVIKKV EKKDK

\section{Cloning of Tetrahymena eEF1A cDNA and site-directed mutagenesis}

Full-length Tetrahymena eEF1A cDNA was obtained by polymerase chain reaction (PCR) amplification of an appropriate clone from a Tetrahymena pyriformis cDNA library (Edamatsu et al., 1991). Primers for amplification (forward [containing a BamHI site], 5'-CGCGGATCCATGGCTAGAGGT-3'; reverse, 5'-TCATTTGTCCTTCTTTTCGACTTTC-3') were designed based on the Tetrahymena eEF1A cDNA sequence (Kurasawa et al., 1992). In Tetrahymena cells, TAA and TAG stop codons are used as glutamine codons (Horowitz and Gorovsky, 1985). There is only one TAG codon in Tetrahymena eEF1A cDNA, which was mutated to CAG by use of a mutated antisense primer, 5'-GCTTCGAATTCACCCTGGGGGGAAGC-3' (the mutated nucleotide is underlined). We used an $E c o R I$ site near the mutation site to ligate the mutated fragment to the rest of the eEF1A sequence to reconstitute a full-length eEF1A cDNA. The ligated DNA fragment was cloned into the $B a m \mathrm{HI}$ and Smal sites of pBluescript II KS. The orientation and sequence of the eEF1A insert was confirmed by the dideoxy chain termination method with an ABI Prism DNA sequencer 377 (Applied Biosystems, California, USA).

\section{Preparation of three GST-domain fusion proteins}

After digestion of the pBlue-Tetrahymena eEF1A construct with $\mathrm{BamHI}$ and $\mathrm{Xhol}$, the DNA fragment was subcloned into the $\mathrm{BamHI}$ and Xhol sites of pGEX-6P-1. By comparison with the sequence for Dictyostelium eEF1A (Yang et al., 1990), we determined the three domains of Tetrahymena eEF1A (Fig. 1A). Insert DNAs for constructing the three GST-domain fusion proteins were prepared by
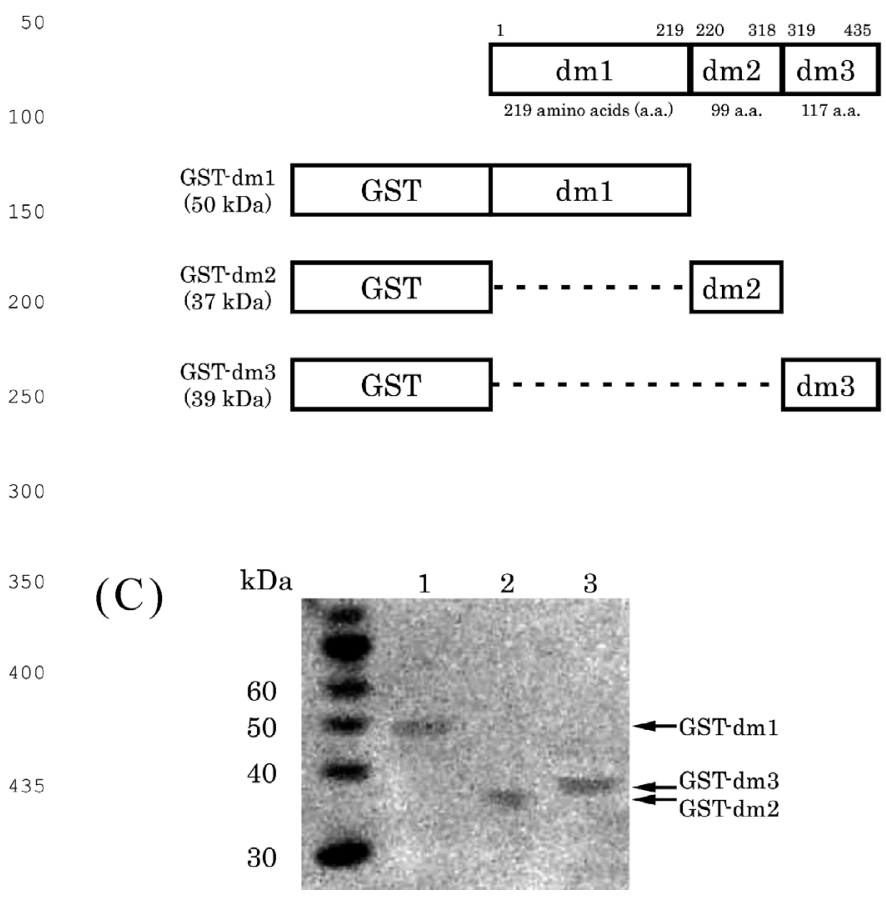

Fig. 1. (A) Amino-acid sequence of Tetrahymena eEF1A. The putative GTP-binding sites and the tRNA-binding site are underlined. The boundaries between the domains ( $\mathrm{dm}$ ) are indicated. The putative GTP-binding sites correspond to the consensus sequences GXXXXGK (a), DXXG (b), and NKXD (c), respectively. The putative tRNA-binding site corresponds to that of $E$. coli EF1A. (B) Schematic diagrams of the structures of GST-dm1, -2, and -3. The three domains of Tetrahymena eEF1A (domains 1, 2, and 3) are shown with their amino-acid residue numbers in parentheses. The calculated molecular masses of GST-dm1, -2, and -3 are 50, 37, and $39 \mathrm{kDa}$, respectively. (C) Purification of GST-dm1, -2, and -3. Proteins were resolved by SDS-PAGE. Molecular mass markers are shown on the left. (1) GST-dm1. (2) GST-dm2. (3) GST-dm3. 
PCR amplification using pGEX-Tetrahymena eEF1A as the template. Forward primers (each containing a $\mathrm{Bg} / \mathrm{ll}$ site) for domain 1 (encoding amino acids 1 through 219), domain 2 (encoding amino acids 220 through 318 ), and domain 3 (encoding amino acids 319 through 435) were 5'-GGAAGATCTGGGATCCCGATGGATAGAGGT-3', 5'-GGAAGATCTGGGATCCCGGCTCTCGATGCT-3' and 5'-GGAAGATCTGGGATCCCGAAGAACGACCCC-3', respectively. Reverse primers (each containing an Xhol site) for domains 1 and 2 were 5'-CCGCTCGAGCTATTCGACGAGGATGGGTCC-3' and 5'-CCGCTCGAGCTAGGCATCGGAAGCGACATT-3', respectively; the reverse primer for domain 3 was the pGEX 3' Sequencing Primer (Amersham Biosciences, Uppsala, Sweden). After digestion with $\mathrm{Bg} / \mathrm{II}$ and $\mathrm{Xhol}$, each DNA fragment was cloned into the $\mathrm{BamH}$ and Xhol sites of pGEX-4T-1. The orientation and sequence of all constructs were verified by automated sequencing using the dideoxy chain termination method.

The three GST-domain fusion proteins were expressed in Escherichia coli strain BL21. Escherischia coli cells harboring each of the constructs were grown in LB medium with $50 \mu \mathrm{g} / \mathrm{ml}$ ampicillin at $37^{\circ} \mathrm{C}$, and the expression of fusion proteins was induced by adding $0.15 \mathrm{mM}$ isopropyl- $\beta$-D-thiogalactosidase for 4 to $6 \mathrm{~h}$ at $25^{\circ} \mathrm{C}$. At the end of induction, the cells were harvested, and the cell pellet was washed once with washing buffer $A(137 \mathrm{mM} \mathrm{NaCl}, 20 \mathrm{mM}$ Tris- $\mathrm{HCl}, \mathrm{pH} 7.5)$ and then resuspended in lysis buffer $(100 \mathrm{mM}$ $\mathrm{NaCl}, 10 \mathrm{mM}$ EDTA, 1\% Triton X-100, $10 \mathrm{mM} \beta$-mercaptoethanol, $10 \mathrm{mg} / \mathrm{ml}$ leupeptin, $10 \mathrm{mM}$ p-toluenesulfonyl-L-lysine chloromethyl ketone, $1 \mathrm{mg} / \mathrm{ml}$ pepstatin $\mathrm{A}, 100 \mathrm{mM}$ phenylmethylsulfonyl fluoride, $20 \mathrm{mM}$ Tris- $\mathrm{HCl}, \mathrm{pH}$ 8.0). After sonication and centrifugation, the supernatant was incubated with Glutathione Sepharose 4B
(Amersham Biosciences, Uppsala, Sweden) at $4^{\circ} \mathrm{C}$ for overnight. The beads were washed with washing buffer $\mathrm{B}(100 \mathrm{mM} \mathrm{NaCl}, 20$ $\mathrm{mM}$ Tris- $\mathrm{HCl}, \mathrm{pH} 8.0$ ), and bound GST fusion proteins were eluted with elution buffer (10 mM glutathione, $50 \mathrm{mM}$ Tris- $\mathrm{HCl}, \mathrm{pH}$ 8.0). The purified GST-domain proteins were analyzed by sodium dodecyl sulfate-polyacrylamide gel electrophoresis (SDS-PAGE).

\section{Preparation of recombinant Tetrahymena CaM}

Recombinant Tetrahymena CaM was expressed as a GST fusion protein in E. coli and purified as described (Hanyu et al., 1995). For GST pull-down assays, GST-Tetrahymena CaM fusion protein was cleaved with thrombin (Amersham Biosciences, Uppsala, Sweden) in thrombin buffer (150 mM NaCl, $2 \mathrm{mM} \mathrm{CaCl}_{2}$, $50 \mathrm{mM}$ Tris- $\mathrm{HCl}, \mathrm{pH} 8.0$ ) at $4^{\circ} \mathrm{C}$. Thrombin then was removed by incubation with Benzamidine Sepharose 6B (Amersham Biosciences, Uppsala, Sweden) at $4^{\circ} \mathrm{C}$, and then recombinant Tetrahymena CaM was dialyzed in MES buffer $(0.75 \mathrm{mM}$ 2-mercaptoethanol, $2 \mathrm{mM}$ $\mathrm{MgCl}_{2}, 40 \mathrm{mM} \mathrm{KCl}, 5 \%$ glycerol, $10 \mathrm{mM}$ 2-[N-morpholino] ethanesulfonic acid, $\mathrm{pH} 7.0)$.

\section{Sequence analysis for putative CaM-binding sites of Tetrahymena eEF1A}

Putative CaM-binding sites of Tetrahymena eEF1A were identified by sequence comparison with the Calmodulin Target Database (http://calcium.uhnres.utoronto.ca/cgi-bin/ctdb/ctdb/home/ $\mathrm{html}$ ). Normalized scores (0 to 9 ) are shown in Fig. 3a. A consecutive string of high values of normalized scores indicates the location of a putative CaM-binding site.
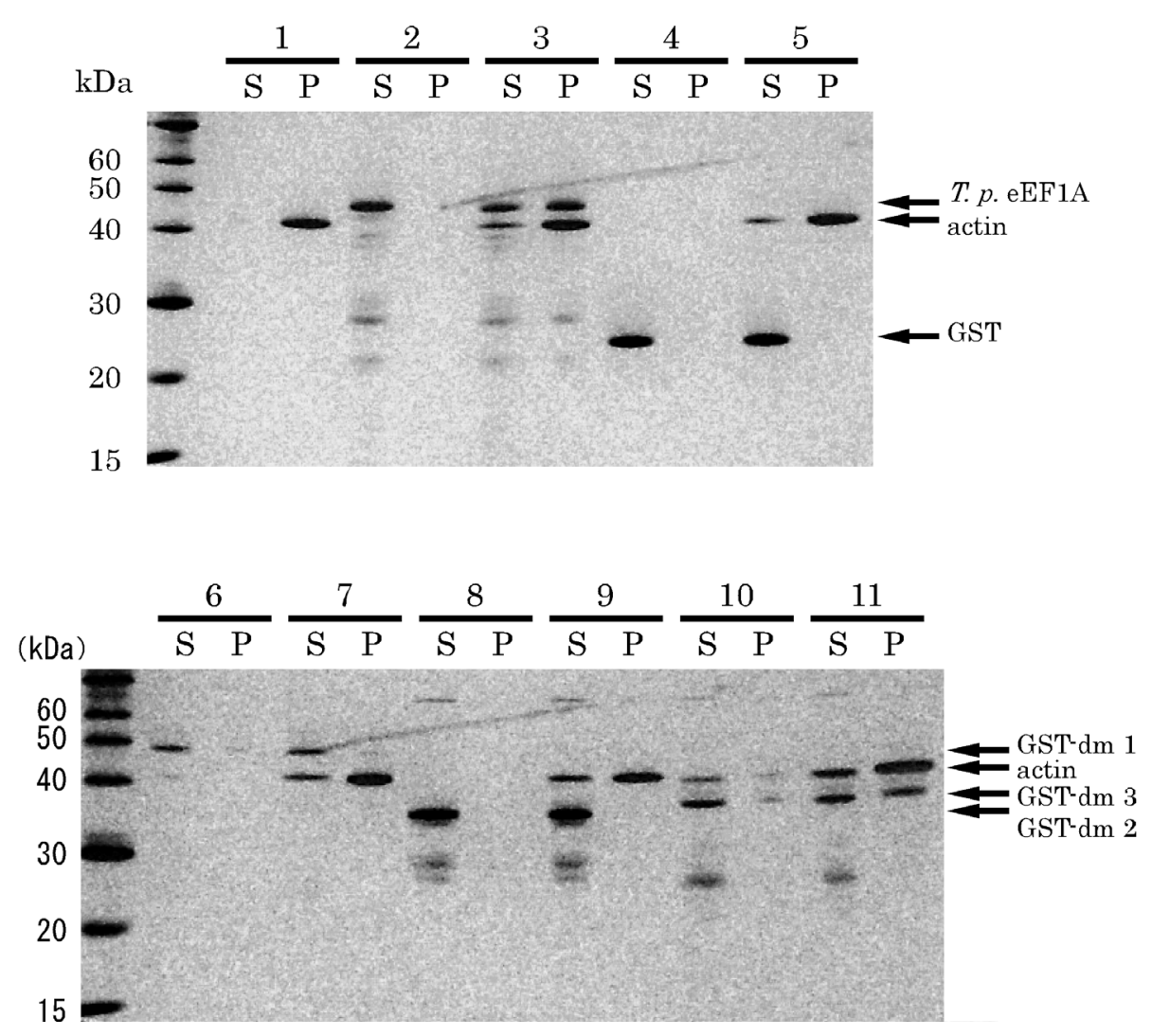

Fig. 2. Binding of GST-dm1, -2 , and -3 to rabbit skeletal muscle F-actin. The supernatants $(S)$ and pellets $(P)$ from the actin cosedimentation assays were analyzed by SDS-PAGE. Bands corresponding to actin, Tetrahymena eEF1A, GST, and GST-dm1, -2 , and -3 are stained with silver and shown by arrows. Molecular mass markers are shown on the left. (1) F-actin alone. (2) Tetrahymena eEF1A alone. (3) F-actin and Tetrahymena eEF1A. (4) GST alone. (5) F-actin and GST. (6) GST-dm1 alone. (7) F-actin and GST-dm1. (8) GST-dm2 alone. (9) F-actin and GST-dm2. (10) GST-dm3 alone. (11) F-actin and GST-dm3. Smaller bands in GST-dm2 and GST-dm3 are probably degradation products. 
(A)

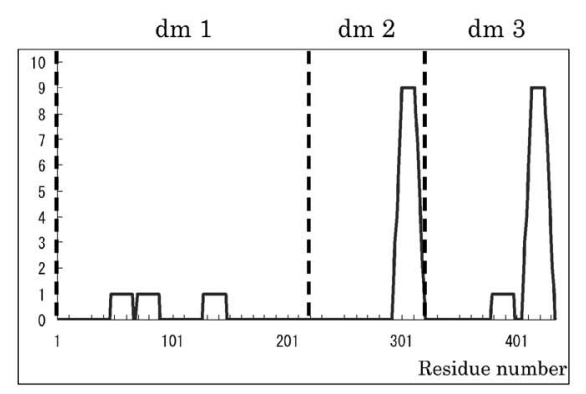

(B)

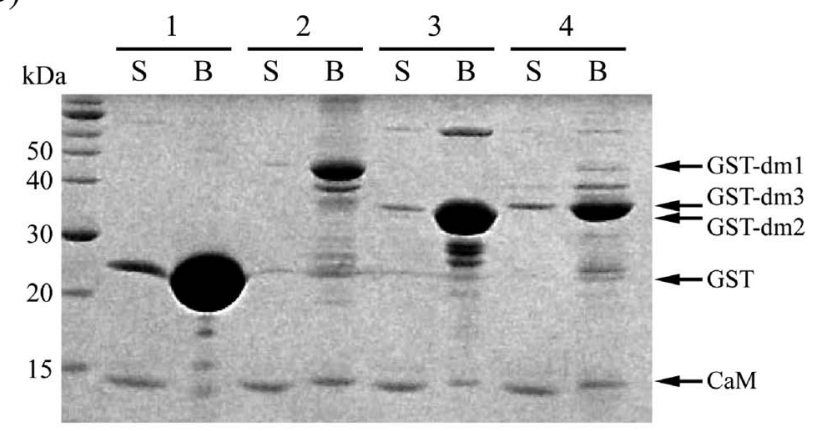

(C)

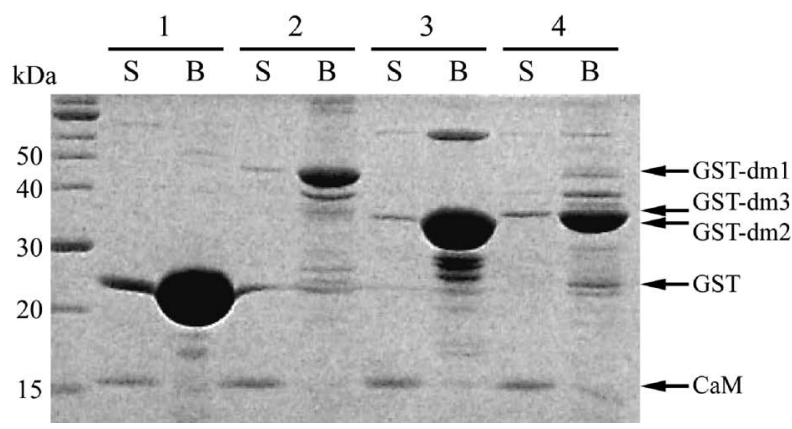

Fig. 3. The putative CaM-binding sites of Tetrahymena eEF1A and binding of GST-dm1, -2 , and -3 to recombinant Tetrahymena CaM with or without $\mathrm{Ca}^{2+}$. (A) Sequence analysis for the putative CaMbinding sites of Tetrahymena eEF1A by the Calmodulin Target Database (http://calcium.uhnres.utoronto.ca/cgi-bin/ctdb/ctdb/home/ $\mathrm{html})$. Vertical axis shows normalized scores (0 to 9$)$ and a consecutive string of high values indicates the location of a putative CaMbinding site. Horizontal axis shows amino acid residue numbers of Tetrahymena eEF1A. The boundaries between the domains are shown by the dotted lines. (B) and (C) GST pull-down assays with GST-dm1 3-bound beads and recombinant Tetrahymena CaM. Assays were performed in MES buffer containing $1 \mathrm{mM} \mathrm{CaCl}_{2}(\mathrm{~B})$ or $1 \mathrm{mM}$ EGTA $(C)$. The supernatants $(S)$ and pellets of beads $(P)$ from GST pull-down assays were analyzed by SDS-PAGE. Bands corresponding to GST-dm1, GST-dm2, GST-dm3, GST, and CaM are shown by arrows. The molecular mass markers are shown on the left. (1) GST-bound beads and CaM. (2) GST-dm1-bound beads and CaM. (3) GST-dm2-bound beads and CaM. (4) GST-dm3bound beads and CaM.

\section{High-speed and low-speed actin cosedimentation assays}

To determine the actin-binding domain(s) of eEF1A, each GSTdomain protein $(2 \mu \mathrm{M})$ in MES buffer was centrifuged at $100,000 \times g$ for $30 \mathrm{~min}$ and the supernatant was mixed with rabbit skeletal muscle F-actin $(2 \mu \mathrm{M})$ in MES buffer. The mixtures were incubated for
$30 \mathrm{~min}$ at $26^{\circ} \mathrm{C}$ and then centrifuged at high speed $(100,000 \times \mathrm{g}$ for $30 \mathrm{~min})$. The supernatants and pellets were analyzed by SDSPAGE.

Because $\mathrm{Ca}^{2+} / \mathrm{CaM}$ inhibits eEF1A-associated $\mathrm{F}$-actin bundle formation, we examined the effect of $\mathrm{Ca}^{2+}$ concentration on the inhibition of F-actin bundle formation. We incubated $3 \mu \mathrm{M} F$-actin, $3 \mu \mathrm{M}$ eEF1A, and $3 \mu \mathrm{M}$ CaM with various concentrations of $\mathrm{Ca}^{2+}$ for 30 $\min$ at $26^{\circ} \mathrm{C}$, and then centrifuged the reactions at low speed $(12,000 \times g$ for $30 \mathrm{~min})$. The supernatants and pellets were analyzed by SDS-PAGE, and the amounts of actin in the supernatants and pellets were determined according to the strength of Coomassie Brilliant Blue R-250 staining of the protein bands.

\section{GST pull-down assay}

To identify the CaM-binding domain(s) of eEF1A, recombinant Tetrahymena CaM $(2 \mu \mathrm{M})$ was incubated with Glutathione Sepharose 4B beads bound to GST or GST-domain fusion proteins in MES buffer containing either $1 \mathrm{mM}, 1 \mu \mathrm{M}$, or $100 \mathrm{nM} \mathrm{CaCl}_{2}$, or 1 $\mathrm{mM}$ EGTA for $30 \mathrm{~min}$ at $26^{\circ} \mathrm{C}$. After centrifugation at $500 \times \mathrm{g}$ for 3 min, the supernatants were removed and the beads were washed three times with MES buffer. The supernatants and the beads were analyzed by SDS-PAGE. To identify the domain(s) involved in dimer formation of eEF1A, Tetrahymena eEF1A $(2 \mu \mathrm{M})$ was incubated with Glutathione Sepharose 4B beads bound to GST or GSTdomain fusion proteins in MES buffer for $30 \mathrm{~min}$ at $26^{\circ} \mathrm{C}$ and analyzed as described above.

\section{Electrophoresis}

SDS-PAGE was performed on $10 \%$ or $15 \%$ running gels with $3 \%$ stacking gels according to the method of Laemmli (1970). Gels were stained with silver or Coomassie Brilliant Blue R-250.

\section{Protein concentration}

Protein concentration was determined by the method of Bradford with bovine serum albumin as a standard (Bradford, 1976).

\section{RESULTS}

\section{Expression and purification of GST fusion proteins}

GST-eEF1A fusion protein was expressed poorly in $E$. coli cells, mostly in the insoluble fraction of total bacterial lysate (data not shown). All three GST-domain constructs were expressed better and were more soluble than the GST-eEF1A fusion protein. However, GST-dm1, -2, and -3 all bound nonspecifically to Glutathione Sepharose 4B beads and were difficult to elute from them. To elute the fusion proteins from the beads, we minimized the volume of beads and prolonged the elution time; with these modifications, we were able to obtain satisfactory amounts of GST$\mathrm{dm} 1,-2$, and -3 . The GST-domain fusion proteins were purified almost to homogeneity (Fig. 1C).

\section{F-actin-binding domain in Tetrahymena eEF1A}

To identify the F-actin-binding domain of eEF1A, we performed cosedimentation assays with GST-dm1, -2, and -3; Tetrahymena eEF1A; and rabbit skeletal muscle F-actin (Fig. 2). In the absence of eEF1A or any of the fusion proteins, most of the F-actin was precipitated by centrifugation, whereas eEF1A, GST, or GST-domain fusion protein alone remained in solution. In the positive control, eEF1A appropriately coprecipitated with F-actin. Similarly, GST-dm3 coprecipitated with F-actin, but GST-dm1 and GST-dm2 did not. Therefore we conclude that only domain 3 of Tetrahymena eEF1A binds to F-actin. 
(A)

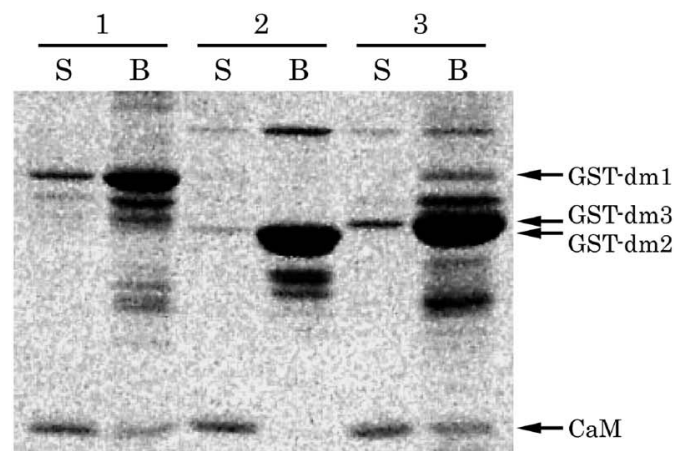

(B)

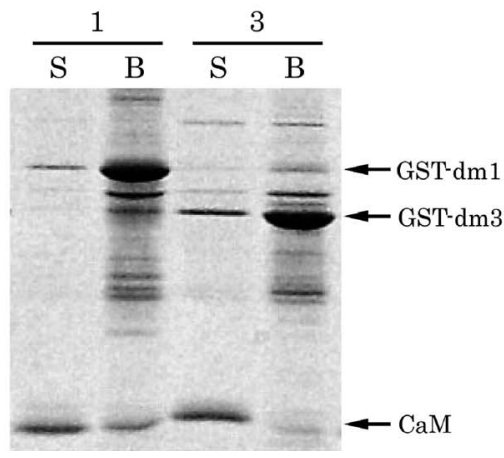

Fig. 4. Binding of GST-dm1, -2 , and -3 to recombinant Tetrahymena CaM at lower $\mathrm{Ca}^{2+}$ concentrations. Assays were performed in MES buffer containing $1 \mu \mathrm{M} \mathrm{CaCl}_{2}$ (A) and $100 \mathrm{nM} \mathrm{CaCl}_{2}$ (B). The supernatants (S) and pellets of beads (P) from the GST pull-down assays were analyzed by SDS-PAGE. Bands corresponding to GST-dm1, GST-dm2, GST-dm3, and CaM are shown by arrows. The molecular mass markers are shown on the left. (1) GST-dm1-bound beads and CaM. (2) GST-dm2-bound beads and CaM. (3) GST-dm3-bound beads and CaM.

\section{CaM-binding domains in Tetrahymena eEF1A}

Using the Calmodulin Target Database, we evaluated putative CaM binding sites in Tetrahymena eEF1A and found that amino acids 301 through 312 and 414 through 425 each had a score of 9 (Fig. 3A). Therefore it seemed likely that both domain 2 (encoding amino acids 220 through 318 ) and domain 3 (encoding amino acids 319 through 435) would bind $\mathrm{CaM}$, whereas domain 1 (encoding amino acids 1 through 219) would not.

To identify the CaM-binding domains of Tetrahymena eEF1A, we performed GST pull-down assays with GSTdm1, -2 , and -3 and recombinant Tetrahymena CaM (Fig. 3B, C). GST-dm1, -2 , and -3 each bound to recombinant Tetrahymena CaM in MES buffer containing $1 \mathrm{mM} \mathrm{CaCl}_{2}$ but not in MES containing $1 \mathrm{mM}$ EGTA. Therefore, all three domains of Tetrahymena eEF1A bind to Tetrahymena CaM in a $\mathrm{Ca}^{2+}$-dependent manner.

To investigate effects of $\mathrm{Ca}^{2+}$ concentration on the binding between each domain and CaM, we repeated the GST pull-down assays at lower $\mathrm{Ca}^{2+}$ concentrations (Fig. 4). Because the concentration of free $\mathrm{Ca}^{2+}$ in Tetrahymena is $100 \mathrm{nM}$ or lower, we evaluated binding at $1 \mu \mathrm{M}$ and $100 \mathrm{nM}$ $\mathrm{CaCl}_{2}$. In MES buffer containing $1 \mu \mathrm{M} \mathrm{CaCl}_{2}$, GST-dm1 and GST-dm3, but not GST-dm2, bound to recombinant

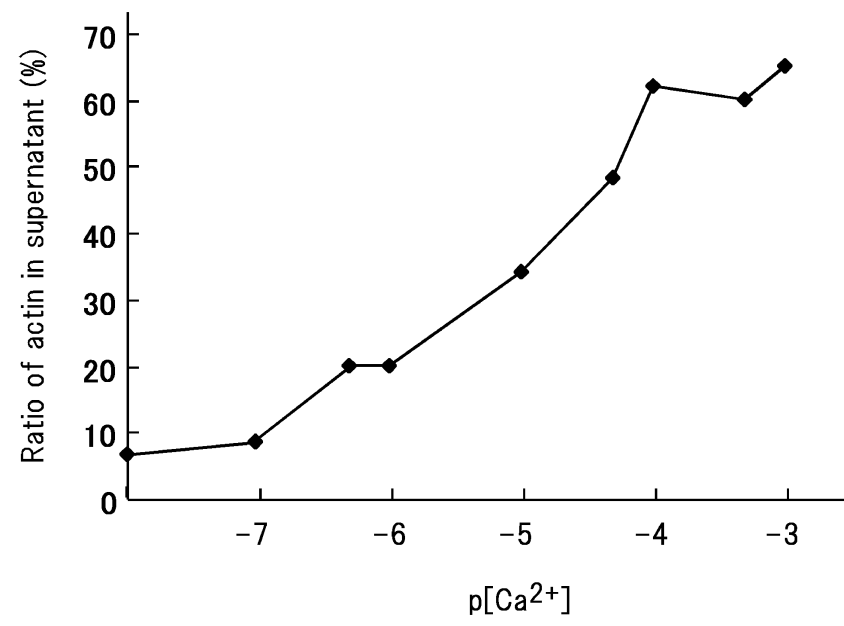

Fig. 5. Inhibition of F-actin bundle formation by $\mathrm{CaM}$ at various $\mathrm{Ca}^{2+}$ concentrations. Cosedimentation assay of $3 \mu \mathrm{M}$ F-actin, $3 \mu \mathrm{M}$ eEF1A, and $3 \mu \mathrm{M}$ CaM was performed at several $\mathrm{Ca}^{2+}$ concentrations $(1 \mathrm{mM}$ EGTA, $100 \mathrm{nM}, 500 \mathrm{nM}, 1 \mu \mathrm{M}, 10 \mu \mathrm{M}, 50 \mu \mathrm{M}, 100 \mu \mathrm{M}, 500 \mu \mathrm{M}$ and 1 $\mathrm{mM} \mathrm{CaCl}$ ). The supernatants and pellets were analyzed by SDSPAGE, and the amounts of actin in the supernatants and pellets were measured from the strength of the Coomassie Brilliant Blue R-250 staining of the protein bands. The $Y$ axis is the ratio of actin in the supernatant to total actin, and the $\mathrm{X}$ axis is the $\mathrm{Ca}^{2+}$ concentration.

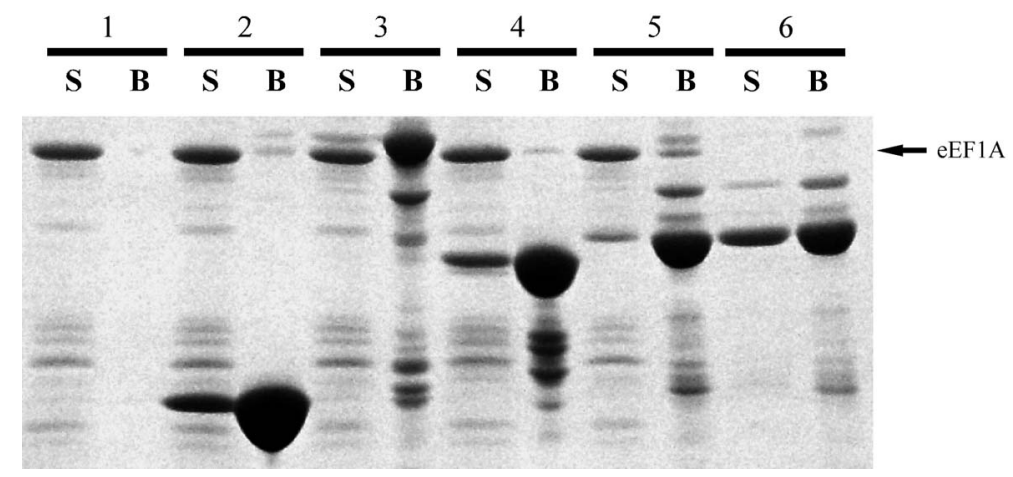

Fig. 6. Binding of GST-dm1, -2 , and -3 to eEF1A in the absence of $\mathrm{Ca}^{2+}$. The supernatants $(\mathrm{S})$ and pellets of beads (P) from GST pull-down assays were analyzed by SDS-PAGE. Bands corresponding to binding of eEF1A to GST-dm3 are shown by an arrow. (1) eEF1A and beads; (2) eEF1A and GST-bound beads; (3) eEF1A and GST-dm1-bound beads; (4) eEF1A and GST-dm2-bound beads; (5) eEF1A and GST-dm3bound beads; (6) GST-dm3-bound beads alone. 

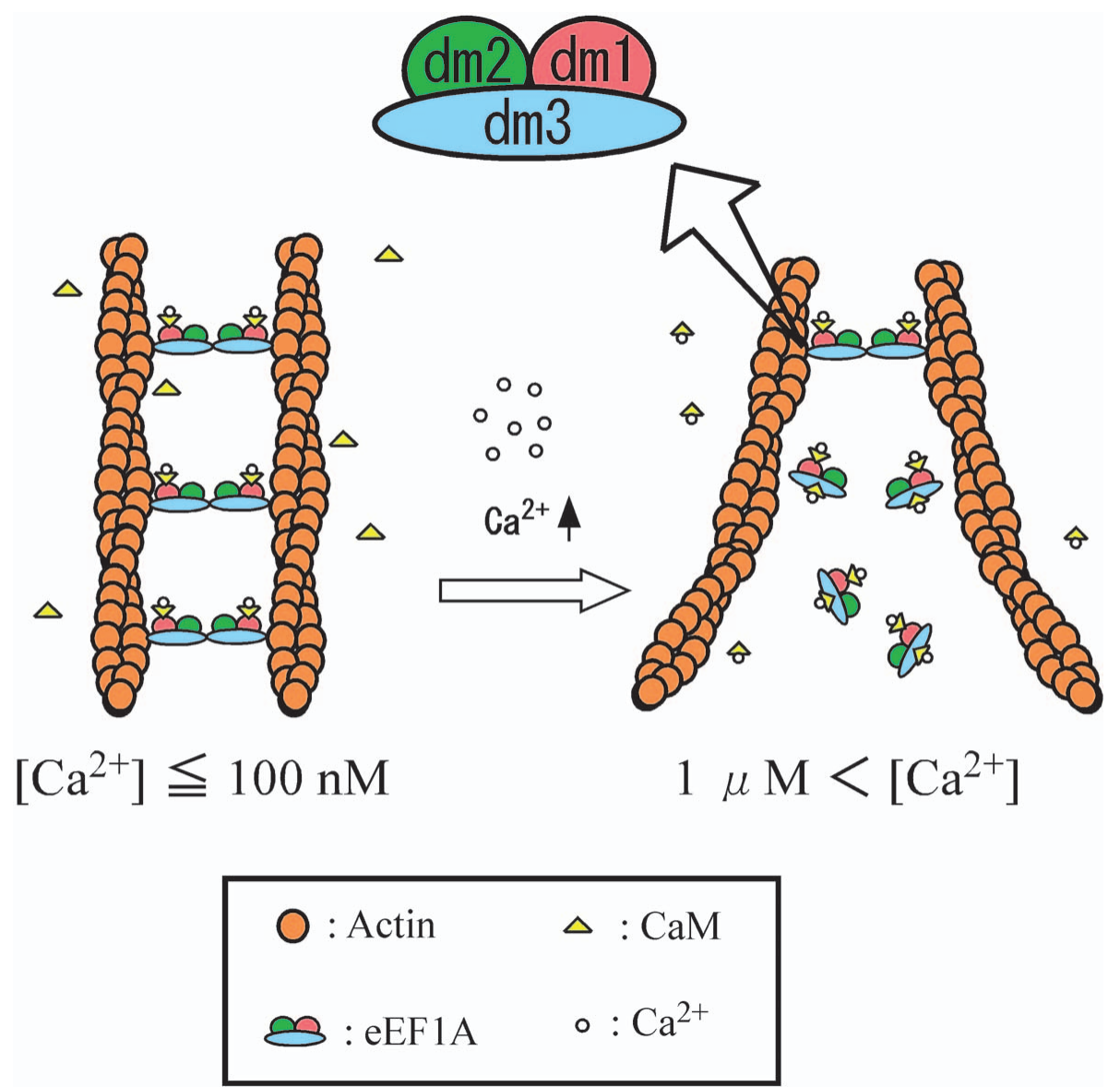

Fig. 7. Schematic illustration of the roles of the three domains of Tetrahymena eEF1A in F-actin bundling. In the presence of $100 \mathrm{nM}$ or less $\mathrm{Ca}^{2+}$, eEF1A forms a dimer through domain 3 and subsequently bundles F-actin. In the presence of $1 \mu \mathrm{M}$ or more $\mathrm{Ca}^{2+}, \mathrm{Ca}^{2+} / \mathrm{CaM}$ binds to domain 3 , a moderate amount of eEF1A dimer separates into monomer, and then $\mathrm{Ca}^{2+} / \mathrm{CaM} / \mathrm{eEF} 1 \mathrm{~A}$ monomer complexes dissociate from $\mathrm{F}$ actin. As a result, F-actin bundles are loosened.

Tetrahymena CaM. Moreover, in MES buffer containing 100 $\mathrm{nM} \mathrm{CaCl} 2$, GST-dm1 bound to recombinant Tetrahymena CaM, and GST-dm3 bound only slightly. These findings indicate that the critical $\mathrm{Ca}^{2+}$ concentration for binding $\mathrm{CaM}$ is $\leq 100 \mathrm{nM}$ for domain 1 , and $100 \mathrm{nM}$ to $1 \mu \mathrm{M}$ for domain 3 .

The F-actin bundling activity of eEF1A is inhibited by $\mathrm{Ca}^{2+} / \mathrm{CaM}$. $\mathrm{Ca}^{2+} / \mathrm{CaM}$ binds one of three domains, and converts eEF1A dimer to monomer, and then F-actin bundles are loosened. At the same time, F-actin filaments are dissociated from the F-actin bundles. To examine which domain is involved in the conversion of eEF1A dimer to monomer, we investigated the effect of $\mathrm{Ca}^{2+}$ concentration on dissociation of $\mathrm{F}$-actin bundles by $\mathrm{CaM}$, using $\mathrm{F}$-actin cosedimentation assays (Fig. 5). At $100 \mathrm{nM} \mathrm{Ca}^{2+}$, F-actin bundles were unaffected. From $500 \mathrm{nM}$ to $1 \mu \mathrm{M} \mathrm{Ca}{ }^{2+}$, F-actin bundles were loosened, and from $1 \mu \mathrm{M}$ to $100 \mu \mathrm{M} \mathrm{Ca}^{2+}$, the amount of F-actin in the supernatant increased. From $100 \mu \mathrm{M}$ to 1 $\mathrm{mM} \mathrm{Ca}^{2+}$, the amount of $\mathrm{F}$-actin in the supernatant reached equilibrium.

\section{Domain for dimer formation in Tetrahymena eEF1A}

To identify the domain involved in the dimerization of eEF1A, we performed GST pull-down assays with GST$\mathrm{dm} 1,-2$, and -3-bound beads and Tetrahymena eEF1A. In the absence of $\mathrm{Ca}^{2+}$ (Fig. 6) as well as in the presence of 1 $\mathrm{mM} \mathrm{Ca}^{2+}$ (data not shown), GST-dm3 bound to Tetrahymena eEF1A, but GST-dm1 and GST-dm2 did not.

\section{DISCUSSION}

This study showed that although all three domains bind to Tetrahymena $\mathrm{CaM}$ in a $\mathrm{Ca}^{2+}$-dependent manner, only domain 3 of Tetrahymena eEF1A binds to F-actin and is involved in the dimerization of eEF1A. Previously, Bunai et al. (2006) demonstrated that eEF1A forms dimers, that dimeric eEF1A bundles F-actin, and that eEF1A monomer binds to but cannot bundle F-actin. Therefore, in Tetrahymena, domain 3 has the most pivotal role in the F-actin bundling activity of eEF1A.

Treatment with $\mathrm{Ca}^{2+} / \mathrm{CaM}$ separated eEF1A dimers into monomers, loosened F-actin bundles, and then dispersed actin filaments (Bunai et al., 2006). Subsequently, $\mathrm{Ca}^{2+} /$ CaM/eEF1A monomer complexes dissociated from actin filaments (Bunai et al., 2006). Therefore, the $\mathrm{Ca}^{2+} / \mathrm{CaM}$ binding site of eEF1A is involved in regulation of the F-actinbundling activity of eEF1A. All three domains bound to Tetrahymena CaM in a $\mathrm{Ca}^{2+}$-dependent manner (Figs. 3, 4). $\mathrm{Ca}^{2+} / \mathrm{CaM}$ inhibition of the F-actin-bundling activity of eEF1A depended on the $\mathrm{Ca}^{2+}$ concentration (Fig. 5). At $100 \mathrm{nM}$ 
$\mathrm{Ca}^{2+}$, domain 1 could bind to CaM but the F-actin bundles were unaffected, suggesting that binding between domain 1 and $\mathrm{CaM}$ has no effect on F-actin bundling by eEF1A. From $500 \mathrm{nM}$ to $1 \mu \mathrm{M} \mathrm{Ca}^{2+}$, domain 3 bound to $\mathrm{CaM}$ but domain 2 did not, and F-actin bundles were partially loosened. These findings suggest that domain 3 has a key role in the $\mathrm{Ca}^{2+} / \mathrm{CaM}$-associated inhibition of $\mathrm{F}$-actin bundle formation. From $1 \mu \mathrm{M}$ to $100 \mu \mathrm{M} \mathrm{Ca}{ }^{2+}$, the amount of F-actin in the supernatant increased with the $\mathrm{Ca}^{2+}$ concentration, suggesting that the binding between domain 3 and CaM increases in a $\mathrm{Ca}^{2+}$-dependent manner. From $100 \mu \mathrm{M}$ to $1 \mathrm{mM} \mathrm{Ca}^{2+}$, the amount of F-actin in the supernatant reached equilibrium. Therefore, the binding between domain 3 and $\mathrm{CaM}$ inhibits F-actin bundle formation by eEF1A.

$\mathrm{CaM}$ has four $\mathrm{Ca}^{2+}$ binding sites, each of which has reasonably high affinity for $\mathrm{Ca}^{2+}\left(K_{a} \sim 10^{-6} \mathrm{M}^{-1}\right)$, rendering $\mathrm{CaM}$ sensitive to transient increases in $\mathrm{Ca}^{2+}$ concentration. Stimulation of and subsequent $\mathrm{Ca}^{2+}$ influx through $\mathrm{Ca}^{2+}$ channels raise the cellular $\mathrm{Ca}^{2+}$ concentration from the basal level of $\sim 100 \mathrm{nM}$ to $\sim 10 \mu \mathrm{M}$. Because of the precise tuning due to $\mathrm{Ca}^{2+}$ affinities, only a few CaM molecules are $\mathrm{Ca}^{2+}$ bound when the cell is in a resting state, but on release of the $\mathrm{Ca}^{2+}$ signal, all CaM molecules are activated. Cooperative binding is particularly important in this context, as it allows for a tightly controlled "all or nothing" response to changes in $\mathrm{Ca}^{2+}$ concentration and, consequently, very clean separation of the "off" and "on" states (Nelson and Chazin, 1998).

However, in the contractile ring, F-actin bundles need to be somewhat loose. If the F-actin bundles in the contractile ring were dispersed completely, the contractile ring would break apart and cytokinesis would cease. If the $\mathrm{Ca}^{2+} / \mathrm{CaM} /$ eEF1A-associated looseness of the F-actin bundles was spread over a large range of $\mathrm{Ca}^{2+}$ concentrations, then the system would be "leaky"- $\mathrm{Ca}^{2+} / \mathrm{CaM}$ would cause some of the eEF1A dimers, which bundle F-actin, to separate into monomers over a large range of $\mathrm{Ca}^{2+}$ concentrations. Therefore, the weak interaction between domain 3 and $\mathrm{Ca}^{2+} /$ $\mathrm{CaM}$ and the $\mathrm{Ca}^{2+} / \mathrm{CaM}$-induced partial separation of eEF1A dimer to monomer leads to looseness of the F-actin bundles in the contractile ring.

In several respects, this study supports Bunai's model, but Bunai's model did not explain how the three domains of eEF1A function in F-actin bundling. From our current results - the roles of domain 3 in F-actin binding, eEF1A dimer formation, and $\mathrm{Ca}^{2+} / \mathrm{CaM}$ regulation of $\mathrm{eEF} 1 \mathrm{~A}-$ we propose a new model of eEF1A-induced F-actin bundling in Tetrahymena cells (Fig. 7). In the presence of $100 \mathrm{nM} \mathrm{Ca}^{2+}$ or less, eEF1A binds to CaM through domain 1, but eEF1A dimerizes through domain 3 . Via domain 3 , the eEF1A both dimerizes and binds F-actin to forms the F-actin bundle. When the $\mathrm{Ca}^{2+}$ concentration increases from $100 \mathrm{nM} \mathrm{Ca}^{2+}$ to $1 \mu \mathrm{M} \mathrm{Ca}^{2+}, \mathrm{Ca}^{2+} / \mathrm{CaM}$ binds to domain 3 and induces a moderate proportion of eEF1A dimer to revert to monomer, subsequently loosening the F-actin bundles. At the same time, binding of $\mathrm{Ca}^{2+} / \mathrm{CaM}$ to domain 3 induces its separation from F-actin, and the $\mathrm{Ca}^{2+} / \mathrm{CaM} / \mathrm{eEF} 1 \mathrm{~A}$ monomer complex subsequently dissociates from F-actin.

\section{ACKNOWLEDGMENTS}

This study was supported by Grants-in-Aid for scientific research from the Japan Society for the Promotion of Science (17657021) to ON.

\section{REFERENCES}

Andersen GR, Pedersen L, Valente L, Chatterjee I, Kinzy TG, Kjeldagaad M, Nyborg J (2000) Structural basis for nucleotide exchange and competition with tRNA in the yeast elongation factor complex eEF1A:eEF1B $\alpha$. Mol Cell 6: 1261-1266

Andersen GR, Nissen P, Nyborg J (2003) Elongation factors in protein biosynthesis. Trends Biochem Sci 28: 434-441

Bradford MM (1976) A rapid and sensitive method for the quantitation of microgram quantities of protein utilizing the principle of protein-dye binding. Anal Biochem 72: 248-225

Bunai F, Ando K, Ueno H, Numata O (2006) Tetrahymena eukaryotic translation elongation factor $1 \mathrm{~A}(\mathrm{eEF} 1 \mathrm{~A})$ bundles filamentous actin through dimer formation. J Biochem 140: 393-399

Durso NA, Cry RJ (1994) A calmodulin-sensitive interaction between microtubules and a higher plant homolog of elongation factor-1 $\alpha$. Plant Cell 6: 893-905

Edamatsu M, Hirono M, Takemasa T, Watanabe $Y$ (1991) The primary structure of Tetrahymena profilin. Biochem Biophys Res Commun 175: 543-550

Edmonds BT, Murray JW, Condeelis $\mathrm{J}$ (1995) $\mathrm{pH}$ regulation of the F-actin binding properties of Dictyostelium elongation factor $1 \alpha$. J Biol Chem 270: 15222-15230

Edmonds BT, Bell A, Wyckoff J, Condeelis J, Leyh TS (1998) The effect of $\mathrm{F}$-actin on the binding and hydrolysis of guanine nucleotide by Dictyostelium elongation factor $1 \mathrm{~A}$. J Biol Chem 273: 10288-10295

Hanyu K, Takemasa T, Numata O, Takahashi M, Watanabe Y (1995) Immunofluorescence localization of a $25-\mathrm{kDa}$ Tetrahymena EFhand $\mathrm{Ca}^{2+}$-binding protein, TCBP-25, in the cell cortex and possible involvement in conjugation. Exp Cell Res 219: 487-493

Horowitz S, Gorovsky MA (1985) An unusual genetic code in nuclear genes of Tetrahymena. Proc Natl Acad Sci USA 82: 2452-2455

Itano N, Hatano T (1991) F-actin bundling protein from Physarum polycephalum: purification and its capacity for co-bundling of actin filaments and microtubules. Cell Motil Cytoskeleton 19: 244-254

Kurasawa $\mathrm{Y}$, Numata $\mathrm{O}$, Katoh M, Hirano H, Chiba J, Watanabe $\mathrm{Y}$ (1992) Identification of Tetrahymena 14-nm filament-associated protein as elongation factor $1 \alpha$. Exp Cell Res 203: 251-258

Kurasawa Y, Hanyu K, Watanabe Y, Numata O (1996a) F-actin bundling activity of Tetrahymena elongation factor $1 \alpha$ is regulated by $\mathrm{Ca}^{2+}$ /calmodulin. J Biochem 119: 791-798

Kurasawa Y, Watanabe Y, Numata O (1996b) Characterization of Factin bundling activity Tetrahymena elongation factor $1 \alpha$ investigated with rabbit skeletal muscle actin. Zool Sci 13: 371-375

Laemmli UK (1970) Cleavage of structure proteins dividing the assembly of the head of bacteriophage T4. Nature 227: 680 685

Liu G, Tang J, Edmonds BT, Murray J, Levin S, Condeelis J (1996) $\mathrm{F}$-actin sequesters elongation factor $1 \alpha$ from interaction with aminoacyl-tRNA in a pH-dependent reaction. J Cell Biol 135: 953-963

Liu G, Grant WM, Persky D, Latham VM, Jr Singer RH, Condeelis J (2002) Interactions of elongation factor $1 \alpha$ with F-actin and beta-actin mRNA: implications for anchoring mRNA in cell protrusions. Mol Biol Cell 13: 579-592

Nelson MR, Chazin WJ (1998) Calmodulin as a calcium sensor. In "Calmodulin and Signal Transduction" Ed by L Van Eldic, DM Watterson, Academic Press, New York, pp 17-64

Pardee JD, Spudich JA (1982) Purification of muscle actin. Methods Enzymol 85: 164-181

Suda M, Fukui M, Sogabe Y, Sato K, Morimatsu A (1999) Overpro- 
duction of elongation factor $1 \alpha$, an essential translational component, causes aberrant cell morphology by affecting the control of growth polarity in fission yeast. Genes Cells 4: 517527

Takeda T, Kurasawa Y, Watanabe Y, Numata O (1995) Polymerization of highly purified Tetrahymena 14-nm filament protein/citrate synthase into filaments and its possible role in regulation of enzymatic activity. J Biochem 117: 869-874
Watanabe Y, Numata O, Kurasawa Y, Katoh M (1994) Cultivation of Tetrahymena cells. In "Cell Biology Vol 1" Ed by JE Celis, Academic Press, [city], California, pp 398-404

Yang F, Demma M, Warren V, Dharmawardhane S, Condeelis J (1990) Identification of an actin-binding protein from Dictyostelium as elongation factor 1a. Nature 347: 494-496

(Received May 25, 2007 / Accepted September 7, 2007) 